\title{
Room tilt illusion and subclavian steal - a case report
}

\author{
Kathrine Arntzen ${ }^{1}$ and Karl B. Alstadhaug ${ }^{1,2^{*}}$ (D)
}

\begin{abstract}
Background: Room tilt illusion (RTI) is a rare symptom of higher vestibular dysfunction, consisting of a transient vertical rotation of the visual scene in the sagittal or coronal plane, most often $90^{\circ}$ or $180^{\circ}$, without any alteration in shape, size and color of objects.

Case presentation: A 63-year-old woman with a history of hypertension and chronic obstructive pulmonary disease went through an uncomplicated aortobifemoral graft surgery due to aortoiliac occlusive disease. Post-operatively she experienced five episodes, lasting from 10 to $30 \mathrm{~min}$, with RTI; $90^{\circ}$ forward rotation of the visual scene in the sagittal plane. Work-up revealed subclavian steal grade 3, and transient ischemia of the central vestibular system of the brainstem was the presumed mechanism.

Conclusion: The course of episodic RTIs is often benign, but RTI may represent ischemia in the posterior cerebral circulation. Both stroke and otoneurologic workup are recommended. To our knowledge, this is the first case of RTI associated with subclavian steal reported.
\end{abstract}

Keywords: Room tilt illusion, Inverted vision, Subclavian steal, Stroke, Case report

\section{Background}

The phenomenon of room tilt illusion (RTI) was first described in a patient thought to suffer from hysteria in 1805 [1]. Relatively few cases have since been reported. In 2012 Sierra-Hidalgo and colleagues presented 13 own cases, and reviewed 135 more that had been previously published [2]. RTI is the perception that the visual scene is transiently tilted $90^{\circ}$ or rotated $180^{\circ}$ (upside-down), and represents a disorder of higher vestibular functions [3], a transient mismatch between the cortical visual and the vestibular three-dimensional (3D) coordinate maps [4]. The underlying cause of RTI varies, from peripheral vestibular [3] and neurological disorders [5] to migraine [6] and stroke [7]. We report a patient with RTI presumed to represent a manifestation of her subclavian steal.

\footnotetext{
* Correspondence: karl.bjornar.alstadhaug@nordlandssykehuset.no 'Department of Neurology, Nordland Hospital, 8092 Bodø, Norway ${ }^{2}$ Institute of Clinical Medicine, The Arctic University of Tromsø, Tromsø, Norway
}

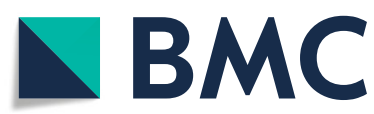

C C The Author(s). 2020 Open Access This article is licensed under a Creative Commons Attribution 4.0 International License, which permits use, sharing, adaptation, distribution and reproduction in any medium or format, as long as you give appropriate credit to the original author(s) and the source, provide a link to the Creative Commons licence, and indicate if changes were made. The images or other third party material in this article are included in the article's Creative Commons licence, unless indicated otherwise in a credit line to the material. If material is not included in the article's Creative Commons licence and your intended use is not permitted by statutory regulation or exceeds the permitted use, you will need to obtain permission directly from the copyright holder. To view a copy of this licence, visit http://creativecommons.org/licenses/by/4.0/ The Creative Commons Public Domain Dedication waiver (http://creativecommons.org/publicdomain/zero/1.0/) applies to the data made available in this article, unless otherwise stated in a credit line to the data.

\section{Case presentation}

A 63-year-old woman was admitted to the hospital due to aortoiliac occlusive disease (Fig. 1). She had a history of hypertension and chronic obstructive pulmonary disease, and was a former smoker for 50 years. Aortobifemoral graft surgery was performed without any major complications, but there was a perioperative bleeding of $1000 \mathrm{ml}$ requiring blood transfusion, plasma and fluids. She was in pain, was subfebrile and was haemodynamically unstable. Norepinephrine, epidural analgesics, oxycodone tablets, antibiotics and fluids were given. In the morning on postoperative (PO) day 1 she reported dizziness, nausea, and a fluctuating "sensation that the room was turned upside-down". It was believed that she was overdosed with opioids, and oxycodone and the epidural were discontinued. On the following day she felt much better and was mobilized, but still experienced 3 more episodes with stereotyped visual illusions, and these recurred twice on PO day 3. All episodes occurred while lying in bed. She was not able to tell whether they were 


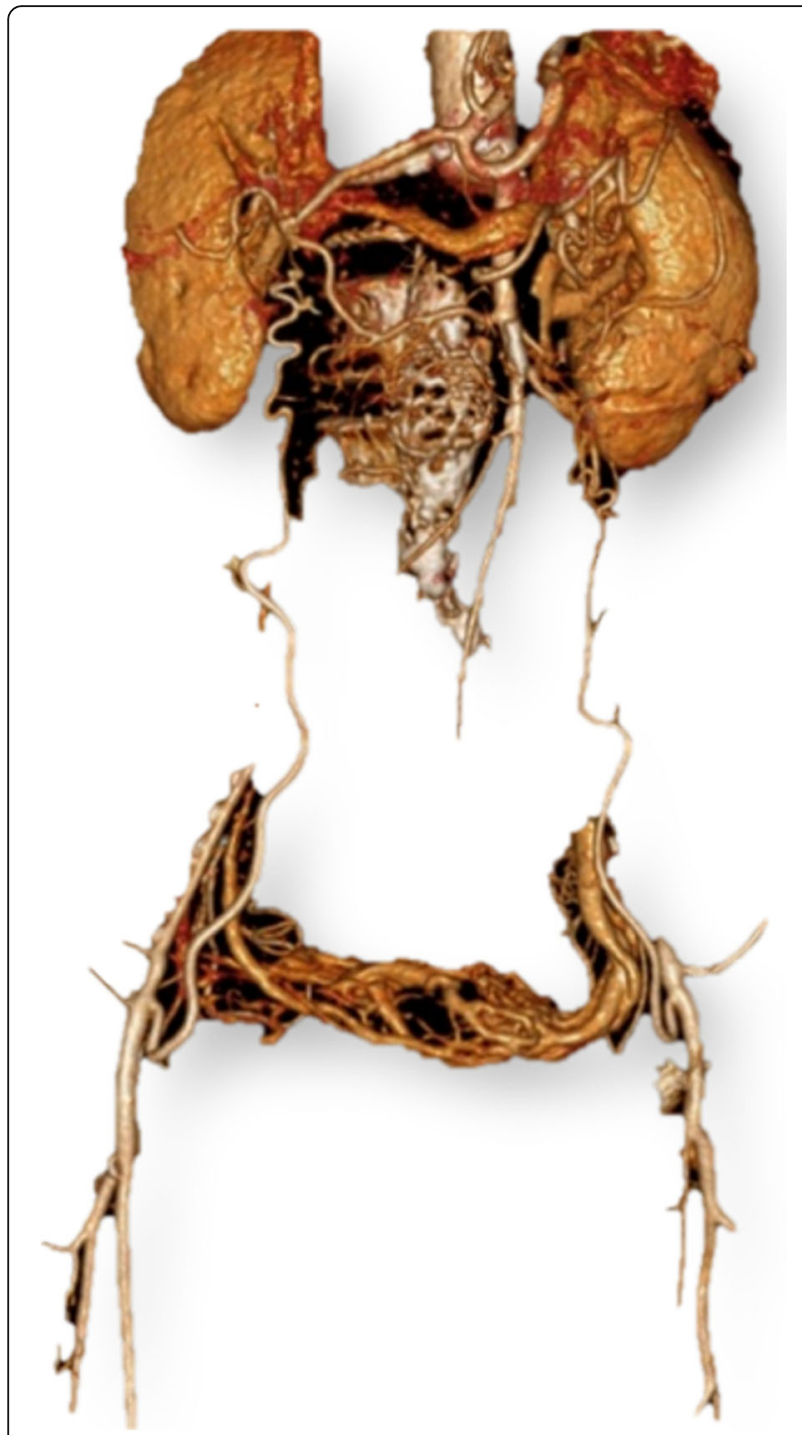

Fig. 1 Three-dimensional digital subtraction CT-angiography demonstrating the patient's abdominal aortic occlusion with collateral blood supply to the legs. (The basic images were obtained with a Philips Ingenuity 128 CT-scanner, and the 3D reconstruction was made by using Intellispace Portal 8.0)

triggered by turning of the head or by the use of her arms. The episodes started suddenly and faded gradually away. Each episode lasted 10 to $30 \mathrm{~min}$. She described unspecific dizziness, but an exact $90^{\circ}$ rotation of the visual scene in the sagittal plane (Fig. 2). She also described a sensation of being translocated to the posterior corner of the ceiling, looking down at the room. No oscillopsia or other associated neurological symptoms were reported. The blood pressure (BP) was not measured during the episodes, and no treatment was given. After the last incident the surgeons asked for a neurological assessment.

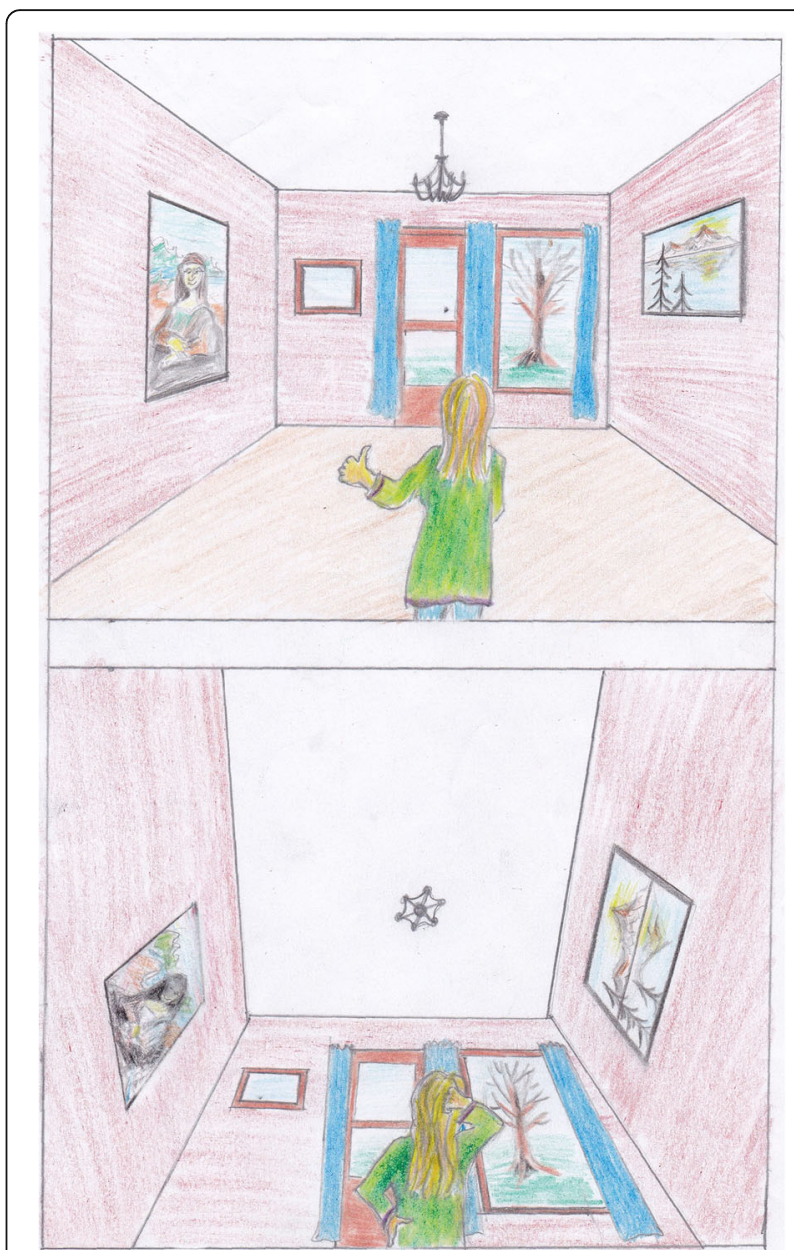

Fig. 2 Cartoon illustrating the visual scene with normal percept and with abnormal percept in RTI with $90^{\circ}$ forward rotation of the visual scene in the sagittal plane. (Drawn by Kristoffer Arntzen)

Work-up with neurological examination and head CT showed nothing amiss, but the BP on her left arm was consistently around $40 \mathrm{mmHg}$ below that on the right arm. Cerebrovascular Doppler Sonography revealed complete retrograde flow from the V4 (Fig. 3a) and the V2 (Fig. 3b) segment of the left vertebral artery (VA), independent of muscular activity of the arm. Besides the standard postoperative care, no specific treatment was given. No more illusions were experienced, and the patient was discharged from hospital. Further diagnostic workup with a head magnetic resonance imaging (MRI) scan was performed 6 weeks later. This revealed an older ischemic lesion in the right external capsule, and absent flow in the left posterior communicating artery (Fig. 4a). The proximal left subclavian artery (Fig. 4b) was occluded. On follow-up 3 months after the discharge from hospital, the patient was in good condition and reporting no neurological symptoms after being discharged from the hospital. A diagnosis of subclavian steal grade 3 

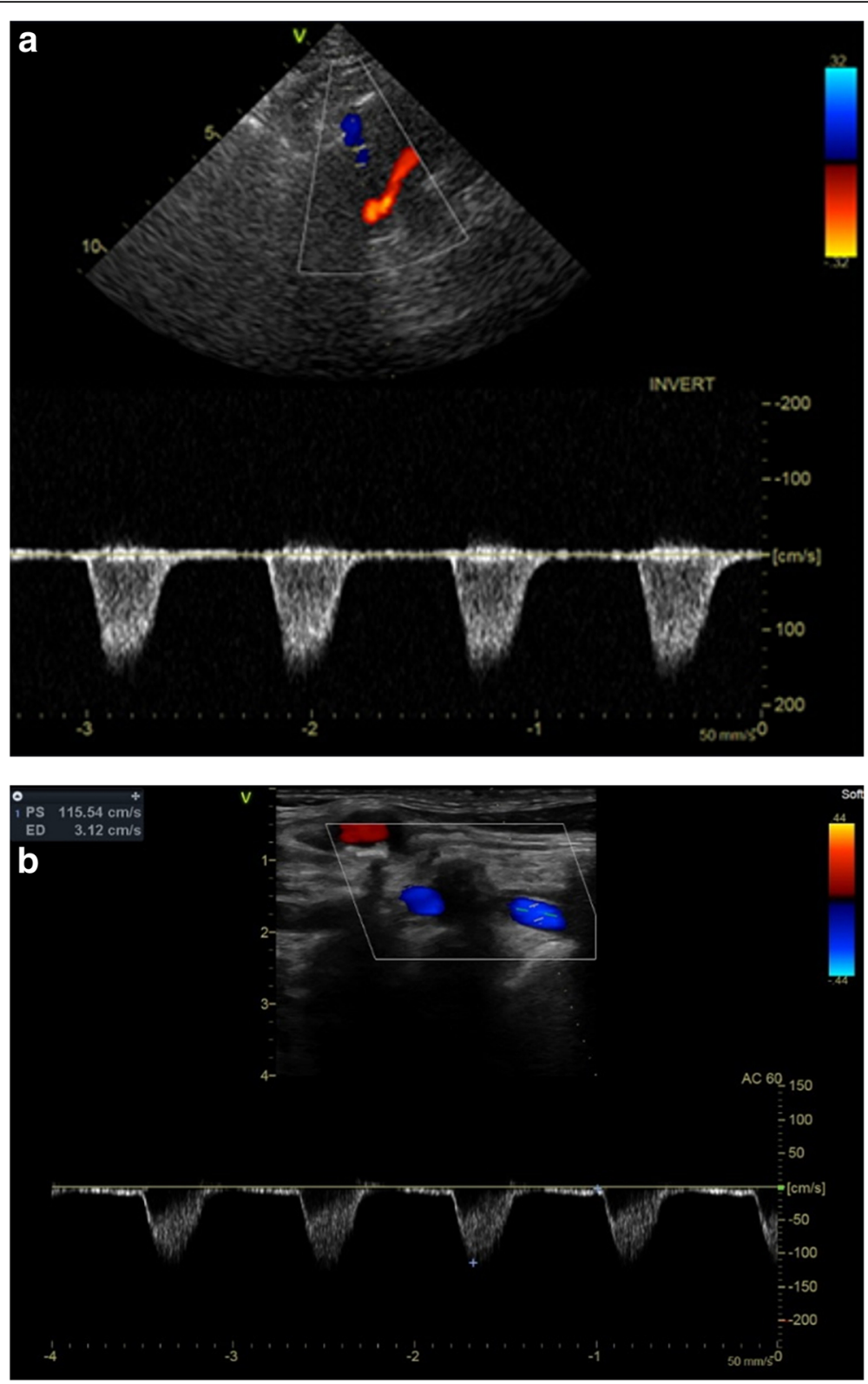

Fig. 3 Diagnostic ultrasound using (a) transcranial color-coded sonography (transforaminal approach) showing retrograde flow in the left V4-VA with velocity $116 \mathrm{~cm} / \mathrm{s}$. $\mathbf{b}$ Extracranial duplex, longitudinal plane, showing complete retrograde flow in the left V2-VA with velocity $116 \mathrm{~cm} / \mathrm{s}$

(permanent retrograde flow in the vertebral artery) was confirmed.

\section{Discussion and conclusions}

When visual illusions are encountered in the clinic, diverse etiologies, which span the fields of psychiatry and neurology, must be considered carefully. The room-tilt illusion may easily be neglected or misdiagnosed if poorly known. In the present case we have described it, to our knowledge for the first time associated with subclavian steal.
Based on their review, Sierra-Hidalgo and colleagues pointed out that ischemic posterior fossa lesions, affecting the vestibular system in particular, probably have been under-recognized, and that the most common documented cause of RTI is posterior circulation stroke [2, 7]. In our patient, only an incidental ischemic brain lesion was found on the head MRI performed weeks after the episodes with RTI, but she was unquestionably at high risk of cerebral ischemia. One can only speculate if early diffusion- weighted MRI (DWI), which is sensitive for early detection of ischemic brain tissue [8], even when transient [9], could have captured an ischemic 

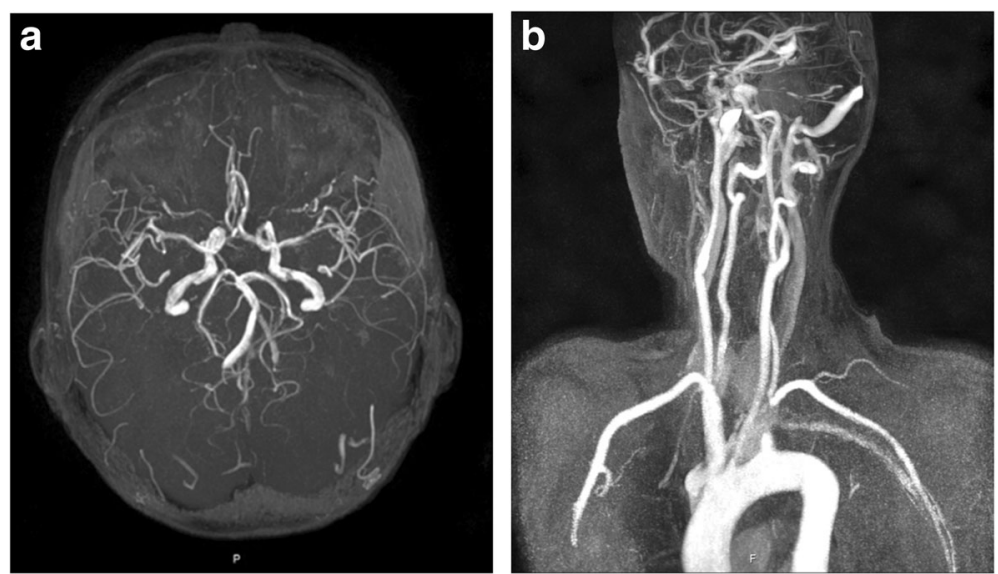

Fig. $4 \mathrm{MR}$ angiography of (a) cerebral arteries showing a missing left posterior communicating artery (b) precerebral arteries showing an occlusion of the proximal left subclavian artery

lesion in close wake of one of her RTIs. Our patient had extensive large vessel disease, and posterior cerebral ischemia was the suspected cause of her RTIs. This presumption led to a diagnosis of subclavian steal.

Subclavian steal (SS) refers to a phenomenon of reversed flow in the vertebral artery ipsilateral to a hemodynamically significant stenosis or occlusion of the prevertebral subclavian artery [10]. In many cases, subclavian steal is asymptomatic, but when causing symptoms due to vertebro-basilar insufficiency or arterial insufficiency of the upper extremity, it becomes a syndrome. Symptoms related to ischemia of the arm are usually not prominent due to the normally slow development of the subclavian artery stenosis with the development of rich collateral supply. On the contrary, transient cerebral symptoms are common. With conventional stroke symptoms from the posterior cerebral circulation, the subclavian steal in our patient would have been considered definitively symptomatic. Still, we find it unlikely that the SS in the present case is coincidental and that alternative mechanisms caused her symptoms. Being somewhat hemodynamically unstable postoperatively probably contributed to minor transient ischemia in the posterior cerebral circulation, manifesting with dizziness and RTI. One could argue that if this was the cause, the RTIs should more likely have occurred when she was in erect rather than in supine position. On the other hand, experimental data have shown that lying horizontally and having a simple visual frame with strongly polarized objects (Fig. 5) increase the risk of forward reorientation illusions [11]. Furthermore, these are usually associated with a levitation illusion. In the present case, the patient experienced the feeling of being lifted to the ceiling during the RTIs, indicating an aberrant inner body image. River and colleagues ascribed the sense of body levitation, an alteration of the body scheme, as a result of dysfunction of the vestibular centers in the brainstem, but also noted that it could represent an epiphenomenon [12].

Traditionally, a parieto-occipital (junction) lesion was considered to be the most common cause of RTI [12], but injury to a variety of locations in both the visual-, vestibular- and the somatosensory system has been

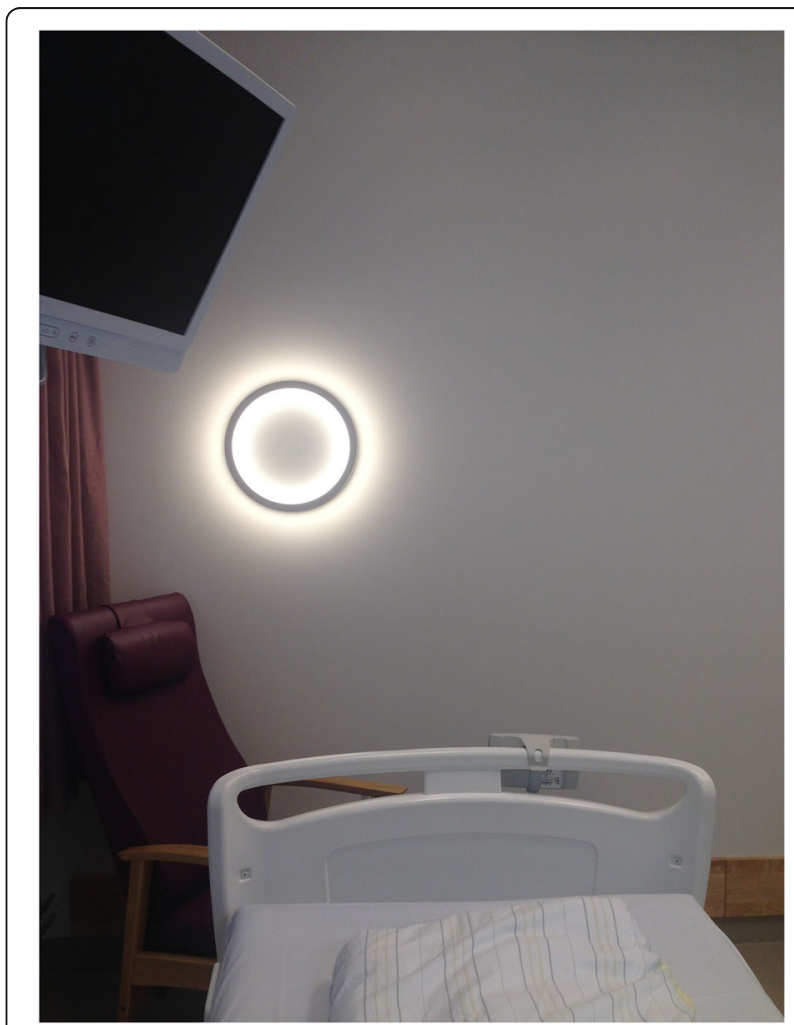

Fig. 5 Picture of the patient's room. Room tilt illusions are probably more likely to occur if the visual frame contains a strongly polarized object 
associated with the phenomenon. This is not surprising since RTI probably includes not only impairment of spatial orientation, but also spatial attention and balance control. As mentioned, the perception of self in space is based on integration of both visual, vestibular and somatosensory input [3].

The lack of detailed physical examinations during the patient's RTIs limit the possibility to draw definite conclusions about the etiology. No otoneurologic workup was performed. Malis \& Guyot reported 23 subjects with RTI that had been assessed at an otoneurologic unit over a 20-year period. All but two were found to have either a vestibular peripheral disorder or a normal assessment finding [13]. All the subjects underwent electronystagmography, and nystagmus was observed in 11. Four without nystagmus were reported to have "vestibular symptoms", and two had positive caloric response. Thirteen subjects underwent MRI, and only one had a pathological finding. No nystagmus was observed in our patient, and she had no story of previous attacks of vertigo, hearing loss or tinnitus.

After our patient's first RTI, delirium caused by opioid intoxication was suspected, but even 2 days after the drug discontinuation she experienced new episodes. Other possible causes of delirium such as infection and withdrawal state were also considered unlikely.

Epilepsy is mentioned as a cause of RTI in the literature, and there is some evidence that vestibular symptoms may occur as a manifestation of focal epileptic activity. To our knowledge, however, no detailed single case report describing RTI strongly associated with epilepsy has been published. No electroencephalography (EEG) was obtained from our patient.

Visual perceptual abnormalities in migraine are common, but illusion of rotation in the frontal plane, as in the present patient, is considered a very rare migraine aura symptom [6]. Our patient had no history of migraine, and experienced no headaches.

In summary, RTI is a transient disorder of the central vestibular system, associated with both peripheral and central lesions. An identifiable cause is often not found, but RTI should not be neglected. It may represent a serious underlying condition, and thorough evaluation is warranted.

\section{Abbreviations}

RTI: Room tilt illusion; PO: Postoperative; BP: Blood pressure; CT: Computer tomography; VA: Vertebral artery; MRI: Magnetic resonance imaging; DWI: Diffusion weighted imaging; SS: Subclavian steal; TIA: Transient ischemic attacks

\section{Acknowledgements}

We would like to thank the patient for her approval of this publication. We would also like to thank Kristoffer Arntzen for contributing with the cartoon illustrating the visual scene in RTI.

\section{Authors' contributions}

KA interviewed, examined and followed up the patient. She wrote the draft of the manuscript. KBA did the ultrasound sonography, gathered relevant information on RTI, contributed intellectually, and revised the manuscript. Both authors read and approved the final manuscript.

\section{Authors' information}

KA is a resident of neurology at the department of neurology, Nordland Hospital. KBA is consultant neurologist at the department of neurology, Nordland Hospital, and professor at the Arctic University of Norway.

\section{Funding}

This study received no funding.

\section{Availability of data and materials}

Clinical data about the patient is stored in her electronic medical journal at Nordland Hospital Trust.

Ethics approval and consent to participate

Not applicable.

\section{Consent for publication}

Written informed consent for publication was obtained from the patient. A copy of the consent form is available for review by the Editor of this journal.

\section{Competing interests}

The authors declare that they have no competing interests.

Received: 7 June 2020 Accepted: 30 September 2020

Published online: 08 October 2020

\section{References}

1. Bishop T. Case of optical illusion from hysteria. Med Phys J. 1805;14:117-8.

2. Sierra-Hidalgo F, de Pablo-Fernandez E, Herrero-San Martin A, CorreasCallero E, Herreros-Rodriguez J, Romero-Munoz JP, et al. Clinical and imaging features of the room tilt illusion. J Neurol. 2012;259(12):2555-64.

3. Brandt T, Dieterich M. The dizzy patient: don't forget disorders of the central vestibular system. Nat Rev Neurol. 2017;13(6):352-62.

4. Brandt T. Cortical matching of visual and vestibular 3D coordinate maps. Ann Neurol. 1997;42(6):983-4.

5. Cochen V, Arnulf I, Demeret S, Neulat ML, Gourlet V, Drouot X, et al. Vivid dreams, hallucinations, psychosis and REM sleep in Guillain-Barre syndrome. Brain. 2005;128(Pt 11):2535-45.

6. Podoll K, Robinson D. Tilted vision, inverted vision, and other forms of rotation. Migraine art. Berkely: North Atlantic Books; 2008. p. 254-7.

7. Pensato U, D'Angelo R, Rinaldi R, Guarino M, Cortelli P. Room tilt illusion and persistent hiccups as presenting symptoms of a left PICA stroke: a case report Neurol Sci. 2019:41(2):469.

8. Kamalian S, Lev MH. Stroke imaging. Radiol Clin N Am. 2019;57(4):717-32.

9. Wardlaw J, Brazzelli M, Miranda H, Chappell F, McNamee P, Scotland G, et al. An assessment of the cost-effectiveness of magnetic resonance, including diffusion-weighted imaging, in patients with transient ischaemic attack and minor stroke: a systematic review, meta-analysis and economic evaluation. Health Technol Assess (Winchester, England). 2014;18(27):1-368 v-vi.

10. Kargiotis $\mathrm{O}$, Siahos $\mathrm{S}$, Safouris A, Feleskouras A, Magoufis G, Tsivgoulis G. Subclavian steal syndrome with or without arterial stenosis: a review. J Neuroimaging. 2016;26(5):473-80.

11. Howard IP, Hu G. Visually induced reorientation illusions. Perception. 2001; 30(5):583-600

12. River Y, Ben Hur T, Steiner I. Reversal of vision metamorphopsia: clinical and anatomical characteristics. Arch Neurol. 1998:55(10):1362-8.

13. Malis DD, Guyot JP. Room tilt illusion as a manifestation of peripheral vestibular disorders. Ann Otol Rhinol Laryngol. 2003;112(7):600-5.

\section{Publisher's Note}

Springer Nature remains neutral with regard to jurisdictional claims in published maps and institutional affiliations. 